\title{
Rearrangement of Noisy Genomes
}

\author{
Chunfang Zheng $^{1}$ and David Sankoff ${ }^{2}$ \\ ${ }^{1}$ Department of Biology, \\ University of Ottawa, Canada K1N 6N5 \\ ${ }^{2}$ Department of Mathematics and Statistics, \\ University of Ottawa, Canada K1N 6N5 \\ \{czhen033, sankoff\}@uottawa.ca
}

\begin{abstract}
Measures of distance between genomic maps are inflated by high levels of noise due to incorrectly resolved paralogy and error at the mapping, sequencing and alignment levels. Comparison is also hampered by lack of information on gene orientation and lack gene order. We suggest a suite of algorithms for genome rearrangement analysis in the presence of noise and incomplete information, and test its robustness as noise levels increase.
\end{abstract}

\section{Introduction}

The comparison of genomic maps is susceptible to high levels of noise that threaten the accuracy and significance of the results. This noise is due to error at the mapping, sequencing and alignment levels, and to incorrect assignment of orthology due to gene, segment or genome duplication. Comparison is also sometimes hampered by lack of gene orientation information and poor resolution of gene order 7]. We suggest an approach to genome rearrangement analysis in the presence of noise and estimate its robustness as simulated noise levels increase.

Our method, designed particularly for unsigned genes (unknown DNA strand) in multichromosomal contexts, is focused on the combinatorial search for the optimal set of compatible "pre-strips". A pre-strip is a set of two or more genes, not necessarily contiguous, on a single chromosome in one genome matched with genes in the same order (or reversed) on any single chromosome in the other genome, i.e., a non-trivial common subsequence of the two chromosomes (one of them possibly reversed). Any set of compatible pre-strips, when all other genes have been deleted from the genomes, becomes a set of "strips"; a strip [5, 4] is a pre-strip with all terms contiguous on the chromosome.

The compilation of pre-strips for our analysis proceeds by an initial identification of all maximal, or inextensible, pre-strips, i.e., pre-strips not contained in another pre-strip, followed by the addition of certain non-maximal pre-strips contained in each maximal pre-strip. We then define a compatibility relation among all these pre-strips, namely whether or nor they can coexist in the same chromosome. This is submitted to a search for a maximum-weight clique (MWC), using the algorithm in [6], where the weights are just the number of genes in the 
pre-strip. Though of course this algorithm may not execute in polynomial time, it is relatively efficient for dense graphs [1], such as the one representing pre-strips for multichromosomal genomes: here, a given pre-strip will not usually involve the same two chromosomes as another pre-strip, and hence will necessarily be compatible with it.

Once the maximal compatible set of pre-strips cum strips is output from the MWC routine, these can be signed by the method in [4, and then submitted to standard rearrangement (GR) algorithms [3, 8, 9,

A large proportion of singletons, i.e., genes not in any pre-strip and hence not considered in the MWC analysis, are nonetheless usually compatible with the output of the MWC and can be reincorporated into the solution, but this must be done judiciously, depending on the origins of the original data.

We test our method on genomes with 100 genes partitioned among four chromosomes, subjecting them to a fixed series of random translocations and inversions before the addition of various levels of noise. Under conditions of moderate noise, the method can assign almost all genes to strips and recover the rearrangements. With increasing noise levels, the distance calculation remains surprisingly stable, though the original rearrangements can no longer be inferred.

In the succeeding sections, we will describe each step as follows:

i) Compile set of all maximal (inextensible) pre-strips. Augment set by adding certain non-maximal pre-strips (Section 2).

ii) Apply MWC [6], with weight = number of genes in a strip (Section 3).

iii) Assign gene orientation according to strip analysis [5, 4] (Section 4).

iv) Input into standard genome rearrangement (GR) algorithm (Section 5).

v) Restoration of singletons (Section 6).

vi) Simulations (Section 7).

Steps i - iii are exact for the problem of maximum weight strip analysis when singletons are disregarded. Step $\mathrm{i}$ is NP-hard, but is tractable for a fixed number of pre-strips, and is very rapid for realistic data. Step ii is NP-hard and is the rate-limiting step in our analysis. Step iii takes polynomial time and is linear if there are no two-term strips in the output of Step ii. Step iv is polynomial. There are several options for Step v. We present exact solutions for three of them.

\section{The Pre-strips}

Because mapping and other errors are likely to result in singleton genes in discordant genomic contexts in the two species being compared, our strategy is to rely entirely on strips to decide which data are erroneous and which are meaningful. But we have to start with structures that are more general than strips in two ways. Intervening error singletons may disrupt a strip, destroying contiguity, or a gene that should be in a strip may be itself erroneously mapped out of the strip, leaving its left and right neighbours adjacent in one of the genomes.

The common order (or its reverse) of the terms of a strip in the two genomes is the only aspect of its structure we have access to. Thus we search for common 


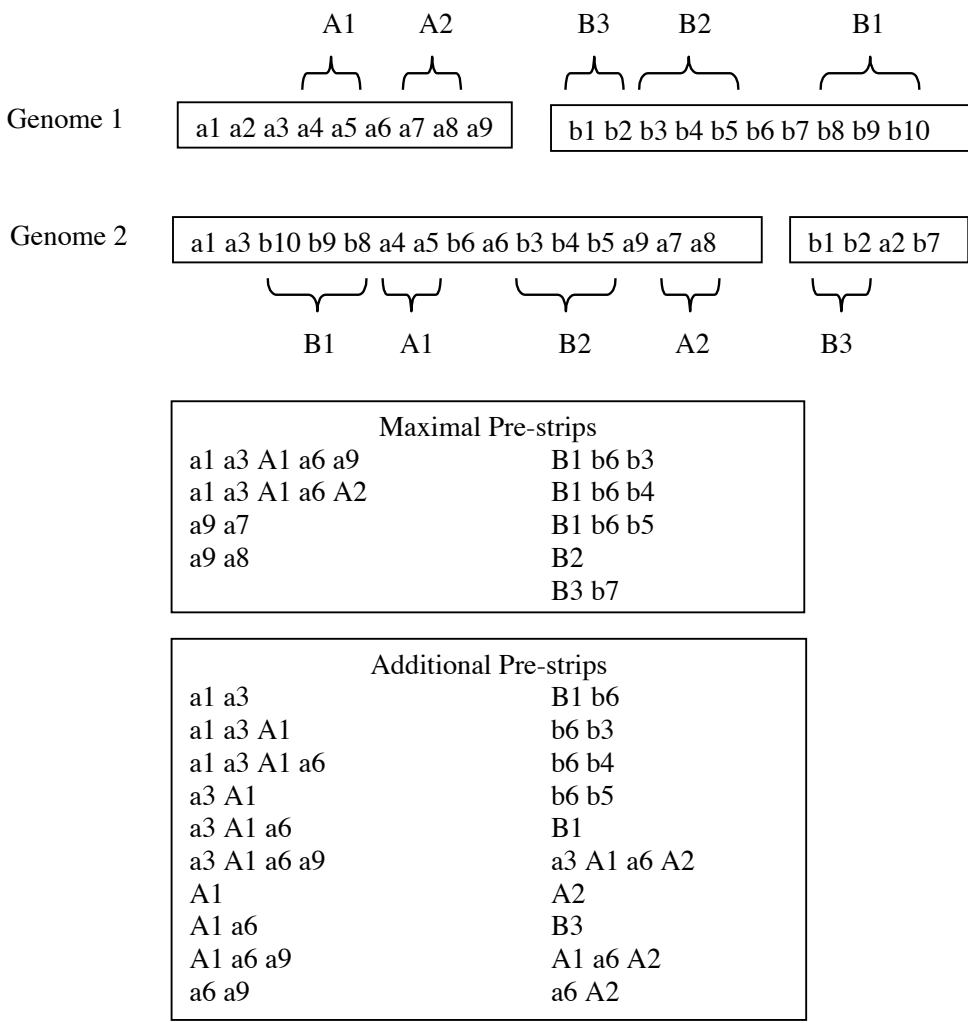

Fig. 1. Example of maximal pre-strips and additional pre-strips to be tested for compatibility. Genomes 1 and 2 both contain two chromosomes. Groups of contiguous genes in both genomes labeled with capital letters. All maximal pre-strips containing A1 intersect with all those containing B1, and are hence incompatible with them. Similarly, the three maximal pre-strips containing a9 are incompatible with each other.

subsequences in the two genomes as potential strips - pre-strips - relying on the subsequent analyses to eliminate the singletons and thus reveal the underlying strips. As a first step then we identify all maximal pre-strips by finding all inextensible common subsequences (and reversed order common subsequences) of the genes in all pairs of chromosomes, one chromosome from each genome. This is easily programmed to run in polynomial time, as long as the number of maximal pre-strips is a fixed parameter, or even when the number of such pre-strips is allowed to grow polynomially as a function of the number of genes.

Our goal is to find strips, i.e., to reduce the pre-strips to strips by eliminating extraneous genes from the chromosome that interrupt the contiguity of pre-strip genes. As shown in Figure1, two maximal pre-strips may intersect, so that both cannot be present in a solution. However all or part of one and part of the other may be present, so that some non-maximal pre-strips must be considered in our search strategy. It suffices to use all contiguous fragments of a maximal pre-strip, 
as illustrated in Figure 1]. (One singleton does not count as a pre-strip, but two singletons, successive terms of a pre-strip, do count.)

We need not consider any other subsequences of maximal pre-strips. To understand this, consider a1 a3 and a6 a9 from maximal pre-strip a1 a3 A1 a6 a9 in Figure 1. If anything intervenes to prevent A1 from forming a strip together with a1 a3 and a6 a9, it will necessarily also prevent a1 a3 and a6 a9 from forming a strip together. Our construction thus establishes the following:

Proposition 1: All possible strips that can be formed by the deletion of genes from two genomes, are contiguous subsequences, containing at least two genes, of inextensible common subsequences (or their reverse) of two chromosomes, one from each genome.

\section{Maximum Weight Cliques}

Once we have a set of pre-strips from which to construct our strips, we next need to construct a matrix of compatibilities among these pre-strips. Two pre-strips are incompatible if they share at least one gene or if either one contains a gene that destroys the contiguity of the genes in the other one. This definition entails:

Proposition 2: Given any set $C$ of pairwise compatible pre-strips. Consider the reduced genomes produced by deleting all genes that are in none of the pre-strips in $C$. In these genomes all of the genes in each pre-strip in $C$ appear as strip.

From the two original $n$-gene genomes, we wish to find a reduction, composed completely of strips, that minimizes the number $d$ of genes deleted. From the compatibility matrix we construct a graph $G$ with the pre-strips as the vertices and with compatible vertices joined by an edge.

Proposition 3: The solution $C$ of the maximum weighted clique problem on $G$, where the weight $w(i)$ on a pre-strip $i$ is simply the number of genes it contains, induces a reduction of the original genomes so that they are composed completely of strips and so that the number of genes deleted is minimized.

Proof: Let $W=\sum_{i \in C} w(i)$. Then the statement follows from Propositions 1 and 2 and

$$
d=n-W .
$$

Kumlander's algorithm [6] is based on a heuristic vertex colouring of sets of independent vertices, followed by two sorts of pruning of the clique search tree, one based on colour classes and the other a backtrack search. Empirically, it was demonstrated to work better than competing algorithms on denser graphs. In the case of genome comparison, we would expect $G$ to become dense, in the sense of 1, as the number of chromosomes increases, as explained in Section 1

In a preliminary implementation of this algorithm, it worked well with hundreds of pre-strips but ran into computing time problems with thousands of vertices.

For the example in Figure 1, the algorithm gives the solution in Figure 2. 


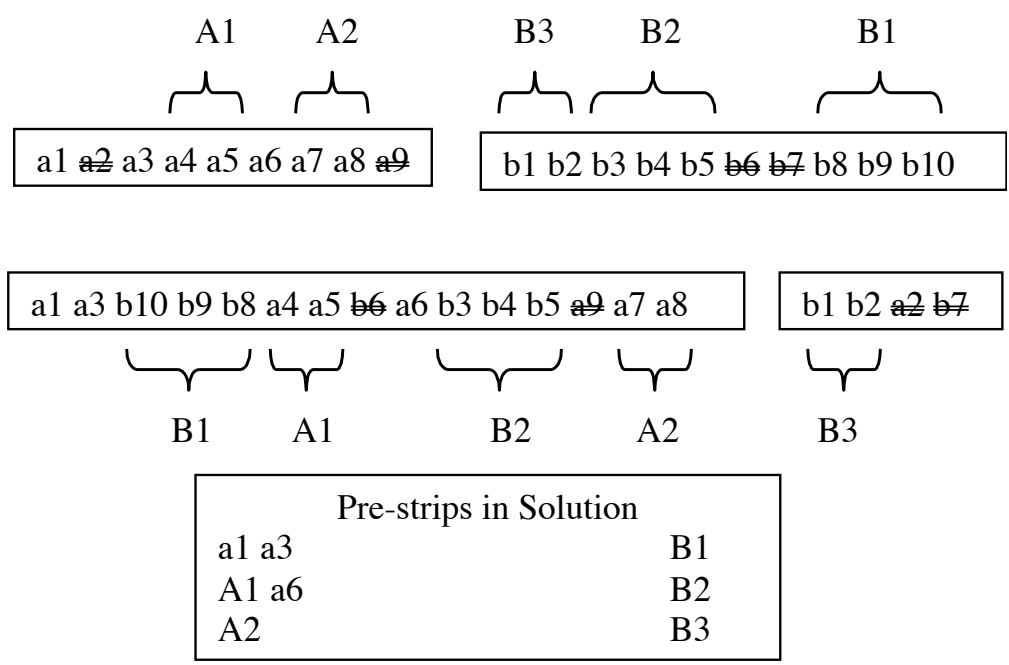

Fig. 2. MWC solution of problem in Figure 1. Rejected singletons a2, a9, b6 and b7 crossed out. Weight $=15$ (out of 19 genes), number of discarded singletons $d=4$.

\section{Sorting Unsigned Genomes}

The problem of adding signs to an unsigned genome so as to a achieve a minimal reversal distance to the identity permutation $1, \cdots, n$ is equivalent to the problem of sorting an unsigned permutation, known to be NP-hard [2].

However, as conjectured in [5] and proved in [4], for all segments of the permutation consisting of three or more consecutive integers (strips) in increasing order, plus signs can be given to all these integers, and for all decreasing strips, such as B1 in the example of Figures 1 and 2, minus signs can be given, and this assignment is consistent with a solution. In [4], it is also shown how to give signs to 2-strips in polynomial time. The algorithm these authors develop is exponential only in $s$, the number of singletons, and is polynomial if $s$ is $O(\log n)$. In our case, however, $s=0$, so sign assignment can be done in polynomial time.

\section{Genome Rearrangement}

To try to infer the rearrangements we used to generate the original data, we constructed all the cycles in the breakpoint graph of the two genomes [3, 9]. A summary statistic is provided by $r=\frac{2(b-c)}{b}$, the reuse rate, where $b$ is the number of breakpoints in the comparison of the two genomes, and $c$ is the number of cycles in their breakpoint graph. The addition of noise generally causes $r$ to increase by adding proportionately more breakpoints than cycles and increasing the sizes of cycles. In the example, $b=5, c=1, d=4$ and $r=1.6$. 


\section{Restoration of Singletons}

There are at least three strategies that can be followed.

1) Restore all singletons that do not conflict with the strips in the MWC. This has the advantages that it is easy to identify these singletons, it includes a maximal amount of the original data in the final analysis and in contexts where the "noise" is due primarily to paralogy or lack of orientation information rather than error, it may help determine relations of orthology and strandedness.

At the same time, it has the disadvantage that in contexts where the "noise" is due primarily to mapping error, it does not reduce this noise to the full extent possible. Moreover, it requires testing all MWCs, which may be numerous in some examples, and for each such clique to check all $2^{s}$ combinations of signs on the $s$ eligible singletons.

With this method, once the singletons are restored, the GR algorithm may return an analysis that differs substantially from that based on the strips only. For example, in Figure 2, genes a2 and b6 conflict with the strips in the solution, and hence cannot be restored to the genomes. At the other extreme, the singleton b7 may be restored without materially affecting the rearrangement inference of three inversions and one translocation. The singleton a9 does not conflict with any strips in the solution, but its inclusion in the GR arrangement changes the solution, requiring an additional inversion. This is a disadvantage only if a9 represents erroneous information. If its singleton status is due to genomic processes, lack of information, or simply insufficient data, the GR analysis may give a more accurate result when such singletons are included.

2) Restore all singletons that do not substantially change the analysis based on the strips only, such as b7. This has the advantage that it only includes items that are maximally compatible with the strip-based analysis, so it should be more effective in excluding noise, while helping resolve lack of orientation. It does increase the amount of the original data included in the final analysis, but this may represent only a small proportion of the singletons. A clear difficulty with this approach is that it requires some operationalization of the notion that two GR analysis based on two different data sets, one subsumed in the other, are substantially the same. For example, we could ask that some optimal GR scenario for the larger set of genes, reduced by omitting all genes absent from the smaller set, also be optimal for the smaller set, taking into account rearrangements that disappear, such as inversions containing genes only in the larger set.

3) Restore no singletons. While this reduces noise maximally, it has the clear disadvantage that it takes into account none of the potentially informative singletons, especially when the noise is not simply mapping error. However, for our artificial (simulated) data, this is the most appropriate method.

We have implemented a program that permits any of these three options, but we present here simulations that test only the final option, generating data that are randomly noisy, without any attempt to model biological or methodological sources of error. Note that a global solution, treating singletons as if they were strips would require a different weighting system for MWC to avoid a trivial solution, and would also have to confront the NP-hardness of GR in this context. 


\section{Simulations}

Prior to our simulations, we divided 100 genes approximately equally among four chromosomes, subjected them to four translocations and 13 inversions, with randomly chosen breakpoints.

For each simulation trial, we add noise by randomly choosing one gene and moving it to a new, random, position in the genome. This models errors such as the mistaken identification of a gene in one genome to be orthologous to one in the other, when it is only a paralog of the true ortholog. We repeat this process, eventually moving a total of 50 genes in a trial. We do 50 such trials in all.

In each trial, we apply our method after each of the 50 random changes and calculate the number of genes in the MWC solution and the distance between the genomes. On the left of Figure 3 we show how the distance remains remarkably stable, despite the loss of over $40 \%$ of the genome to noise. More telling, we calculate the reuse rate ( $2 \mathrm{x}$ distance / number of breakpoints), a measure of how random one genome is with respect to the other, and thus how much doubt should be thrown on the genome rearrangement analysis. The right side of Figure 3 shows a steady increase in reuse, eventually approaching 2.0, the maximum value, indicative of complete randomness. Although the distance remains more or less constant, the rearrangements inferred between the two genomes change drastically as noise is added. Thus while our method seems to infer genome distance in a robust way, the details of the rearrangement scenario are obscured by increased noise levels.
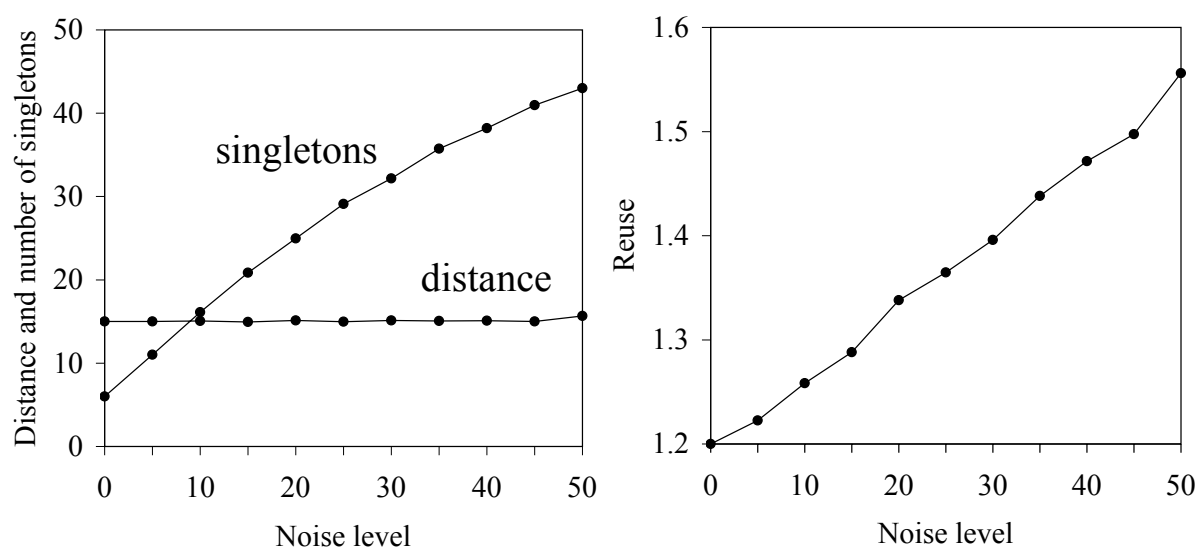

Fig. 3. Left: stability of genome distance (inversions plus translocations) despite the loss of large numbers of singletons. Right: increase in reuse as a result of noise.

\section{Conclusions}

The main contribution of this work is the conversion of the maximal weight strip problem to the MWC problem, based on the induced elimination of as 
few genes as possible from the genomes being compared. Our method recovers the underlying rearrangements while the noise level is low, but is degraded by higher levels. In our simulations, the total genomic distance, though not the actual rearrangements, remain fairly constant despite high levels of noise.

Though our pre-strip computation is not worst-case polynomial-time, in real problems it is not the bottleneck. Nevertheless, we are now implementing a polynomial time algorithm for producing only 2- and 3- strips. Applying the MWC algorithm to these small strips, followed by piecing all overlapping ones together, gives the same solution as the present method.

The MWC itself which is the bottleneck. Since our compatibility graph is dense, methods such as those introduced in 1 might speed up the MWC search.

It might be thought that with the advent of genome sequencing, comparative mapping without strandedness would become obsolete. In fact, the trend towards low-coverage sequencing without finishing, leaving many gene order ambiguities, makes it likely that physical and genetic mapping methods will continue to predominate, aside from very few model organisms.

\section{Acknowledgements}

Research supported in part by grants from the NSERC (Canada). DS holds the Canada Research Chair in Mathematical Genomics and is a Fellow of the Evolutionary Biology Program of the Canadian Institute for Advanced Research.

\section{References}

1. Arora, S., Karger, D. and and Karpinski, M. 1999. Polynomial time approximation schemes for dense instances of NP-hard problems. JCCS 58:193-210.

2. Caprara, A. 1997. Sorting by reversals is difficult. RECOMB '97. 75-83.

3. Hannenhalli, S. and Pevzner, P.A. 1995. Transforming cabbage into turnip (polynomial algorithm for sorting signed permutations by reversals). STOC 27. 178-189.

4. Hannenhalli, S. and Pevzner, P.A. 1996. To cut or not to cut (applications of comparative physical maps in molecular evolution). SODA 7. 304-313.

5. Kececioglu, J. and Sankoff, D. 1993. Exact and approximation algorithms for the inversion distance between two permutations. CPM 4, LNCS 684. 87-105.

6. Kumlander, D. 2005 A new exact algorithm for the maximum-weight clique problem based on a heuristic vertex-coloring and a backtrack search. Fourth European Congress of Mathematics. Poster and manuscript.

7. Sankoff, D., Lenert, A. and Zheng, C. 2005. Reversals of fortune. RECOMB 2005 Ws on Comparative Genomics, LNBI 3678. 131-141.

8. Tesler, G. 2002. Efficient algorithms for multichromosomal genome rearrangements. JCSS 65:587-609.

9. Yancopoulos, S., Attie, O. and Friedberg, R. 2005. Efficient sorting of genomic permutations by translocation, inversion and block interchange. Bioinformatics 21: 3340 - 3346 . 